\title{
A98-39896
}

\section{AIAA-98-4951 TOPOLOGY OPTIMIZATION FOR FLEXTENSIONAL ACTUATORS}

\author{
S. Nishiwaki, E C. N. Silva, Y. Li and N. Kikuchi \\ Department of Mechanical Engineering and Applied Mechanics, University of Michigan,
}

Ann Arbor, 3005 EECS, MI,48109-2125

\begin{abstract}
Flextensional actuators consist of a piezoceramic device and a flexible mechanical structure which converts and amplifies the output piezoceramic displacement. In this research, a methodology to design flextensional actuators with high output displacement and large generative forces is proposed based upon topology optimization techniques and finite element method (FEM). The problem is posed as the design of a flexible mechanical structure coupled with piezoceramic which has the maximum displacement in some specified directions due to an input of electrical charge in piezoceramic. Low frequency and static applications are considered. The optimal solution meets both kinematic requirement and structural requirement. The numerical implementations were done by using Sequential Linear Programming(SLP). Some 2-D topologies of flextensional actuator are presented for different applications.
\end{abstract}

\section{INTRODUCTION}

Flextensional actuators consist of a piezoceramic device and a flexible mechanical structure. Piezoceramic has the property to convert electrical energy into mechanical energy, and vice versa. Flexible mechanical structures are designed to deform in a specified direction when boundary traction is applied.

The performance of flextensional actuators is measured in terms of output displacement and generative force (or "blocking") force ${ }^{1}$. Generative force is the maximum force supported by the transducer without deforming, for a certain applied voltage. Simple analytical models, experimental techniques ${ }^{2}$ and finite element method ${ }^{1}$ have been used to develop flextensional actuators. However, the design is limited to the optimization of some dimension of a specific topology chosen for coupling structure. These studies have shown that the generative force and output displacement are a pair of design trade-off and depend on the topology of the coupling structure which decides the distribution of stiffness and flexibility. Therefore, designs of flextensional actuator can be achieved by design of coupling structure topology using topology optimization.

\footnotetext{
* Copyright $(\mathcal{1} 1998$ by the American Institute of Aeronautics and Astronautics, Inc. All rights reserved.
}

Based on this idea, in this research we proposed a design method for flextensional actuator by applying topology optimization techniques and finite element method. The problem is posed as the design of a flexible structure coupled to the piezoceramic that maximizes the output displacement and generative force in some specified direction. Since only the static and low frequency applications of these actuators will be considered, we do not take account dynamic effects in this work. The homogenization design method which was first developed by Bends $\varnothing \mathrm{e}$ and $\mathrm{Kikuchi}^{3}$ is utilized as a topology optimization method. FEM is applied to the structural analysis in the optimization procedure. Even though two-dimensional (plane strain) topologies of flextensional actuators are presented as illustration, the method can be extended to three-dimensional cases.

\section{TOPOLOGY OPTIMIZATION PROCEDURE}

\section{Homogenization Design Method}

The topology optimization problem is formulated as a problem of finding the optimal distribution of material properties in a extended fixed domain that maximizes some structure cost function. Therefore the finite element model does not change during the optimization process. This technique is applied based on homogenization method.

To relax the optimization problem, a microstructure proposed by Bends $\varnothing \mathrm{e}$ and Kikuchi ${ }^{3}$ is defined in each point of the domain which consists of a unit cell with a rectangular hole inside (See Fig. 1). The use of microstructure allows the appearance of intermediate (or composite) materials rather than only void or full material in the final solution. The design variables are the dimensions $\alpha, \beta$ and the orientation $\theta$ of the microstructure hole. In this sense, the problem is to optimize the material distribution in a perforated domain with infinite microscope voids. At each point of the domain a microstructure defines a composite material. The effective properties of these composite materials, which is called homogenized properties are obtained 


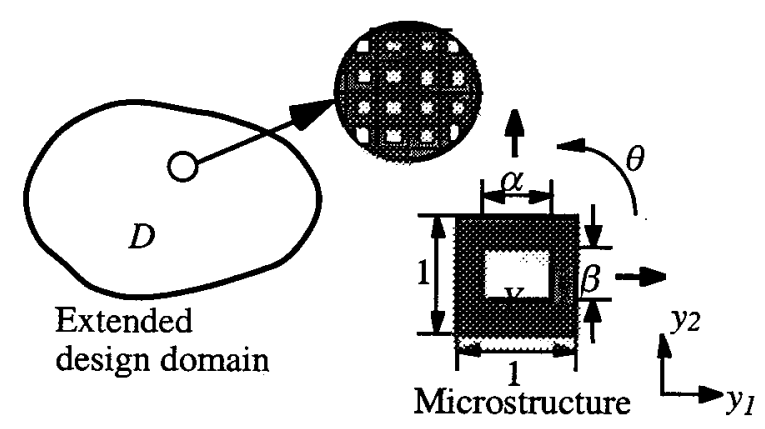

Figure. 1: Microstructure in the homogenization design method

using homogenization method.

\section{Formulation of Optimization Problem}

Two important requirements must be considered in the flextensional actuator design: the kinematic requirement and the structural requirement. For kinematic requirement, the flextensional structure must have maximum deformation along a direction specified by a dummy load $t_{2}$ at when surface charge $q_{1}$ is applied at boundary $\Gamma_{q_{1}}$ as shown in Fig. 2(a). The function for the kinematic requirement is obtained by the extended mutual mean compliance or the mean transduction posed by traction $\boldsymbol{t}_{2}$ at boundary $\Gamma_{t_{2}}$ and the displacement field $u_{1}$ due to surface charge $q_{1}{ }^{4}$. It is given by expression:

$$
\begin{aligned}
L_{2}\left(\boldsymbol{u}_{1}, \phi_{1}\right) & =L_{t}\left(\boldsymbol{t}_{2}, \boldsymbol{u}_{1}\right) \\
& =A\left(\boldsymbol{u}_{1}, \boldsymbol{u}_{2}\right)+B\left(\phi_{1}, \boldsymbol{u}_{2}\right)+B\left(\phi_{2}, \boldsymbol{u}_{1}\right)-C\left(\phi_{1}, \phi_{2}\right) \\
& =L q\left(q_{1}, \phi_{2}\right) \\
& =B\left(\phi_{2}, \boldsymbol{u}_{1}\right)-C\left(\phi_{1}-\phi_{2}\right)
\end{aligned}
$$

where:

$$
\begin{aligned}
& A(\boldsymbol{u}, \boldsymbol{v})=\int_{\Omega} \varepsilon(\boldsymbol{u})^{t} c^{E} \varepsilon(\boldsymbol{v}) d \Omega \\
& B(\phi, v)=\int_{\Omega}(\nabla \phi)^{t} e^{t} \varepsilon(\boldsymbol{v}) d \Omega \\
& C(\phi, \varphi)=\int_{\Omega} \nabla \phi^{t} \varepsilon^{S} \nabla \varphi d \Omega \\
& L_{t}\left(\boldsymbol{t}_{i} \cdot \boldsymbol{v}_{i}\right)=\int_{\Gamma_{t_{i}}} t_{i} \cdot v_{i} d \Gamma \\
& L_{q}\left(q_{i}, \varphi_{i}\right)=\int_{\Gamma_{q_{1}}} q_{i} \varphi_{i} d \Gamma
\end{aligned}
$$

and $\Omega$ is the fixed design domain (it contains both piezoelectric materials and elastic materials), $\nabla$ is the gradient operator, $\varepsilon()$ is the strain operator, $t_{i}$ is a traction vector, $q_{i}$ is the surface electrical charge and $c^{E}, e$ and $\varepsilon^{S}$ are the elastic, piezoelectric and dielectric properties, respectively, of the medium. $\boldsymbol{u}$ and $\nu$ are displacements $\phi$ and $\varphi$ are electric potentials.
For the structural requirement, the flexible structure must have sufficient stiffness at boundary $\Gamma_{t_{2}}$ in order to maintain the shape of flexible structure against the reaction force imposed by the workpiece, as shown in Fig. 2(b). The function for structural requirement is obtained by minimizing the mean compliance at boundary $\Gamma_{t_{2}}$ posed by traction $-t_{2}$, while the electric potential is set to be zero at boundary $\Gamma_{q_{1}}$. It is given by expression ${ }^{4}$ :

$$
L_{3}\left(u_{3}, \phi_{3}\right)=\int_{\Gamma_{t_{2}}} t_{3} \cdot u_{3} d \Gamma=A\left(u_{3}, u_{3}\right)+B\left(\phi_{3}, u_{3}\right)
$$

Since the maximization of the mean transduction and minimization of the mean compliance have a tradeoff relation, and all of the Pareto optima may not provide the appropriate structural configuration. Thus we formulate new multi-objective functions to obtain an appropriate optimal solution incorporating functions for both requirements from Pareto optima. It is proposed as $^{5}$ :

$$
F(\mathrm{x})=\frac{L_{2}\left(\boldsymbol{u}_{1}, \phi_{1}\right)}{L_{3}\left(\boldsymbol{u}_{3}, \phi_{3}\right)}
$$

Therefore the new optimization problem is stated as:

$$
\begin{aligned}
& \text { Maximize: } F(\mathrm{x}) \\
& \alpha, \beta \text { and } \theta
\end{aligned}
$$

Subject to: Equilibrium conditions

$$
\begin{aligned}
& 0 \leq \alpha \leq \alpha_{\text {sup }}<1 \\
& 0 \leq \beta \leq \beta_{\text {sup }}<1 \\
& \Theta(\alpha, \beta)=\int_{S}(1-\alpha \beta) d S-\Theta_{S} \leq 0
\end{aligned}
$$

where $S$ is the design domain $\Omega$ excluding the piezoceramic, $\Theta$ is the volume of this design domain and $\Theta_{S}$ is an upper bound volume constraint to control the volume constraint to control the maximum amount of material to build the coupling structure.

Since the finite element discretization was applied to the design domain, the problem was solved in its discretized form. The design variables $\alpha, \beta$ and $\theta$ which theoretically are continuous functions of $x$, became variable sets $\alpha_{n}, \beta_{n}$ and $\theta_{n}$ corresponding to $n$ finite elements. The upper bounds $\alpha_{\text {sup }}$ and $\beta_{\text {sup }}$ were specified for $\alpha$ and $\beta$ respectively to avoid singularities of the stiffness matrix in the finite element formulation (due to the presence of "null" material in the domain). Numerically, the region with $\alpha=\beta=0.995$ have no structural significance and can be regarded as void. So the upper bounds $\alpha_{\text {sup }}$ and $\beta_{\text {sup }}$ were chosen to be 


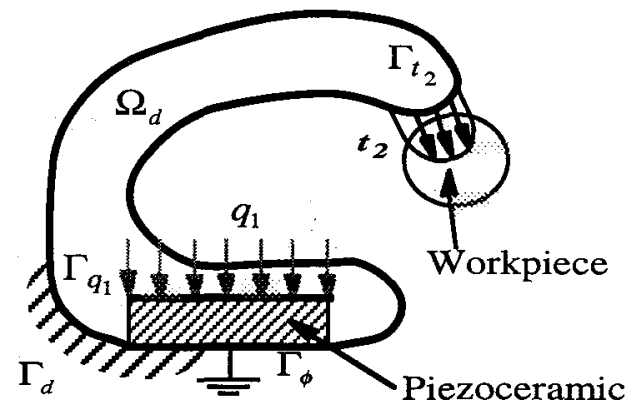

Case (a)

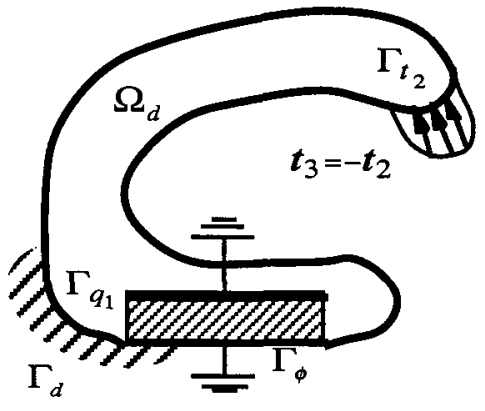

Case (b)

Figure 2: Load cases for calculation of mean transduction (a) and mean compliance(b)

0.995 in this research.

\section{Numerical Implementation}

Fig. 3 shows a flowchart of the optimization procedure. Sequential linear programming (SLP) was used as an optimizer. It consists of the sequential solution of linearized problem defined by writing a Taylor series expansion for the objective and constraint functions around the current design points $\alpha_{n}$ and $\beta_{n}$ in each iteration step. $\theta_{n}$ was obtained by considering the directions of local principal stresses in each finite element after each optimization step. The sensitivities of the objective functions for the need of linearization were derived in Silva ${ }^{4}$.

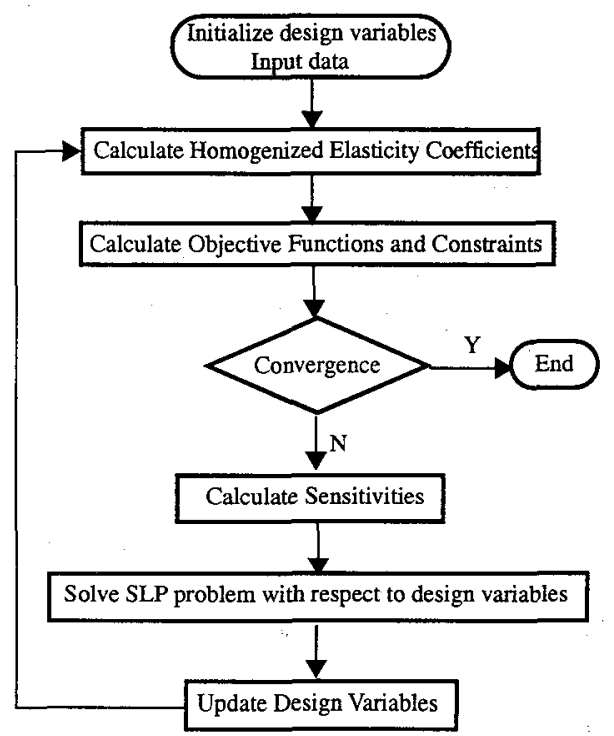

Figure 3: Flowchart of Optimization Procedure

The initial value of the microscopic design variables $\alpha_{n}, \beta_{n}$ and $\theta_{n}$ were chosen to be $0.9,0.9$ and 0.0 respectively. There were moving limits defined in each iteration, which allowed design variables to change within $5-15 \%$ of their original values. After one optimization step, a new set of design variables $\alpha_{n}$ and $\beta_{n}$ was obtained and updated in the design domain.

\section{RESULTS}

Unlike our previous research, where axial symmetric actuators were considered as examples, some unsymmetrical two-dimensional topologies of flextensional actuators will be shown to illustrate the method described above. The design domain consists a piezoceramic domain and a domain of brass (elastic material) where the optimization is conducted. The domain is discretized as a finite element model. Mechanical and electrical boundary conditions are applied. Electrical degrees of freedom are considered only in the piezoceramics domain. For each example, the corresponding interpretation of the topology obtained using image based techniques described in Kikuchi et al. $^{6}$, is also presented.

Table 1 describes the piezoelectric material properties used in the simulation. The Young's modulus and Poission's ratio of the brass are $106 \mathrm{GPa}$ and 0.3 respectively. Two-dimensional plane strain elements were used in the finite element analysis. For all examples, the total volume constraint $\Theta_{S}$ was chosen to be $20 \%$ of the volume of the brass domain (S).

Table 1: Material Properties of PZT5

\begin{tabular}{c|c}
\hline Material Constants & PZT5 \\
\hline$c_{11}^{E}\left(10^{10} \mathrm{~N} / \mathrm{m}^{2}\right)$ & 12.1 \\
\hline$c_{12}^{E}\left(10^{10} \mathrm{~N} / \mathrm{m}^{2}\right)$ & 7.54 \\
\hline
\end{tabular}


Table 1: Material Properties of PZT5

\begin{tabular}{l|l}
\hline Material Constants & PZT5 \\
\hline$c_{13}^{E}\left(10^{10} \mathrm{~N} / \mathrm{m}^{2}\right)$ & 7.52 \\
\hline$c_{33}^{E}\left(10^{10} \mathrm{~N} / \mathrm{m}^{2}\right)$ & 11.1 \\
\hline$c_{44}^{E}\left(10^{10} \mathrm{~N} / \mathrm{m}^{2}\right)$ & 2.30 \\
\hline$c_{66}^{E}\left(10^{10} \mathrm{~N} / \mathrm{m}^{2}\right)$ & 2.10 \\
\hline$e_{13}\left(\mathrm{C} / \mathrm{m}^{2}\right)$ & -5.4 \\
\hline$e_{33}\left(\mathrm{C} / \mathrm{m}^{2}\right)$ & 15.8 \\
\hline$e_{15}\left(\mathrm{C} / \mathrm{m}^{2}\right)$ & 12.3 \\
\hline$\varepsilon_{11}^{S} / \varepsilon_{0}$ & 1650 \\
\hline$\varepsilon_{33}^{s} / \varepsilon_{0}$ & 1700 \\
\hline
\end{tabular}

\section{Example 1:}

The initial domain considered for this example is described as in Fig. 4. The optimization problem is defined as the maximization of the deflection at point $\mathrm{A}$ in the horizontal direction when electrical charge $q_{1}$ is applied at the top surface of the piezoceramic block. The coupling structure obtained using the proposed method is shown in Fig. 5.

Finite element simulation of the result design was conducted to verify the function of the actuator. The commercial FEA package ABAQUS was used with those material constants described earlier in this session. As shown in Fig. 6, the deformed structure has significant deflection in the desired direction.

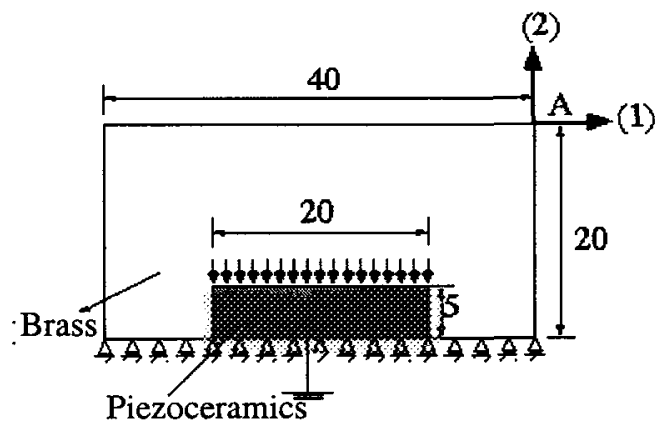

Figure 6: Design Domain Considered for Example 1(1) and Example 2(2)

\section{Example 2:}

For the second example, we consider the same design domain as in example 1 , while the objective is to

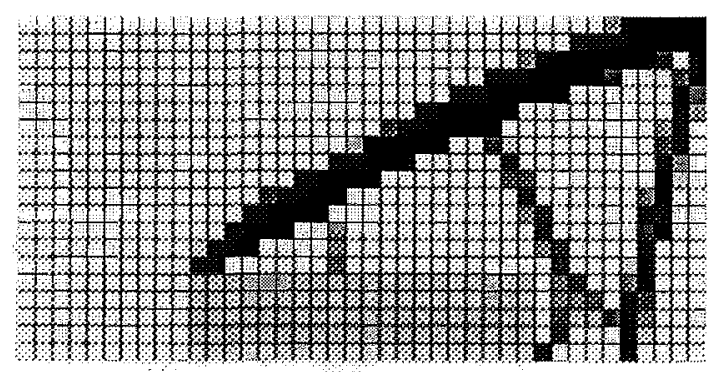

(a) Optimal Configuration

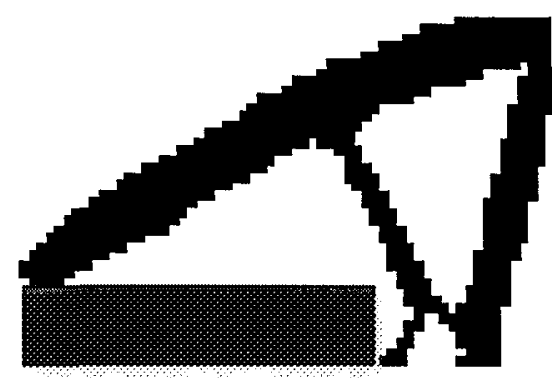

(b) Image

Figure 5: Optimal topology obtained for Example 1

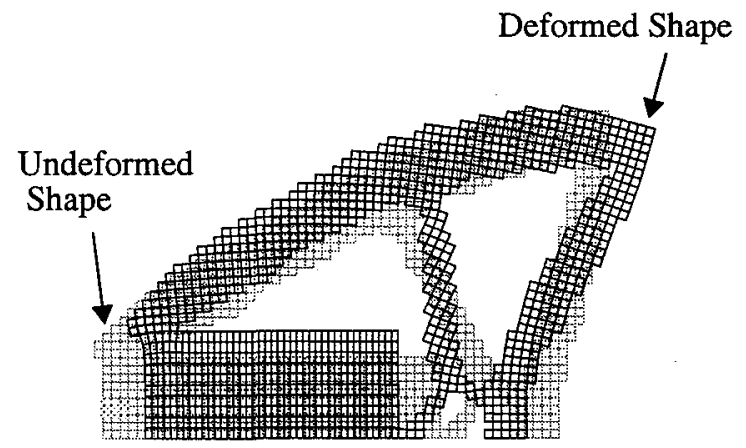

Figure 6: Deformed Configuration Obtained From ABAQUS simulation

maximize the deflection of point $\mathrm{A}$ in the vertical direction. The design result is shown in Fig. 7 along with the image processed interpretation.

\section{Example 3: Torsion Actuator}

Up to now, most actuator designs we considered are single output actuators. However, our optimization program can deal with multiple output case as well. Fig. 8 shows a square design domain with a smaller square piezoceramic material in the center. The objective is to maximize the deflections at each corner of the square in 


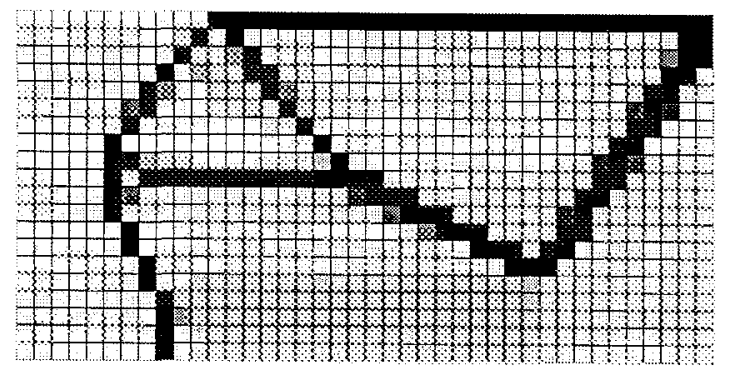

(a) Optimal Configuration

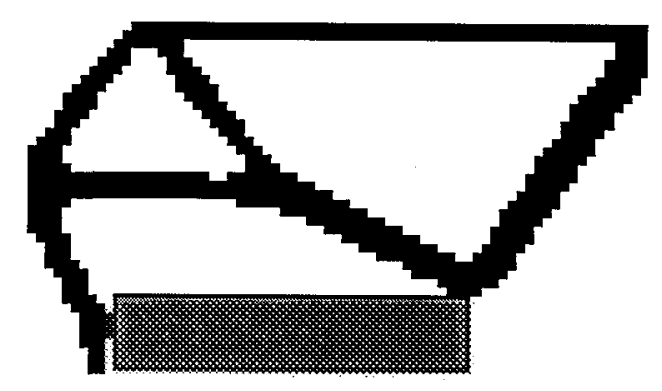

(b) Image

Figure 7: Optimal Topology for Example 2

the specified directions (as shown in Fig. 8). Electric charge is charged at the top surface while the bottom surface is connected to the ground. The center line of piezoceramic block is constrained from moving vertically and the middle point is completely fixed.

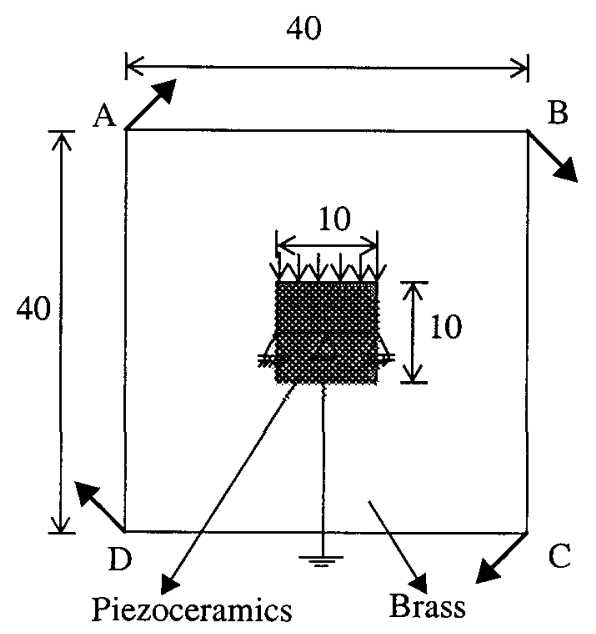

Figure 8: Design Domain Considered for Example 3 (Torsional Actuator)

Therefore, we can regard this design as a actuator which produce torsion or torque. This type of actuators has wide industrial applications. The design result

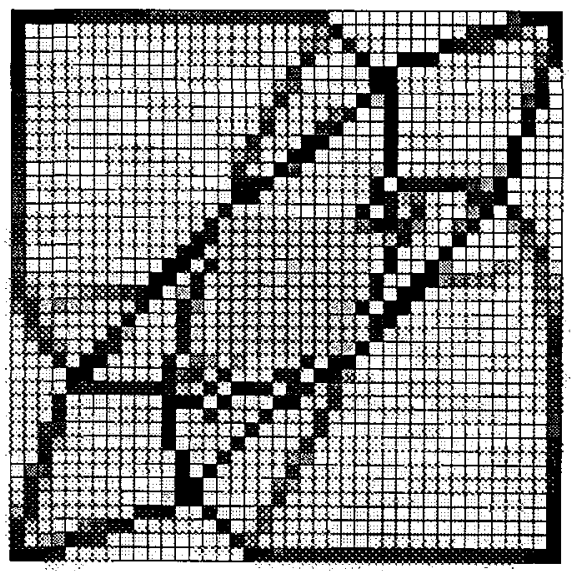

(a) Optimal Configuration

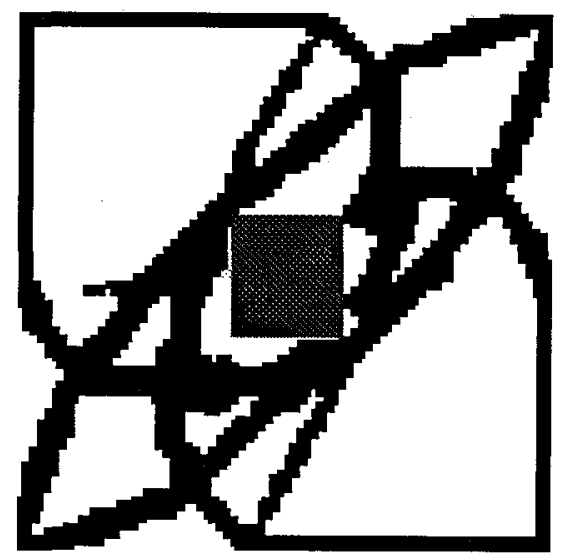

(b) Image

Figure 9: Optimal Topology for Torsional Actuator

reveals an symmetry property of the solution as show in Figure 9

\section{Conclusion}

A method of flextensional actuator design for static and low-frequency applications has been proposed. This method is based upon the homogenization design method for optimal topology design. The design problem is formulated as the topology optimization of the flexible structure connected to a unchanged piezoceramic domain to amplify and convert the output piezoceramic displacement. The examples presented illustrated different applications and finite element simulation verified the result. Furthermore, even though not included in this paper, the design can be extended to 3-D actuator design. The complex topologies obtained can be manufactured using rapid prototyping techniques. 


\section{References}

1. Dogan, A., Uchino, K., and Newnham, R. E. (1997)

Composite Piezoelectric Transducer with Truncated Conical Endcaps "Cymbal" IEEE Transactions on Ultrasonics, Ferroelectrics and Frequency Control 44 No. 3 May 597-605.

2. Dogan, A., Yoshikawa, S., Uchino, K., and Newnham, R.E. (1994) The Effect of Geometry on the Characteristics of the Moonie Transducer and Reliability Issue Proceedings of IEEE 1994 Ultrasonic Symposium 935-939

3. Bends $\varnothing$ e, M. P. and Kikuchi, N. (1988) Generating Optimal Topologies in Structural Design Using a Homogenization Method Computer Methods in Applied Mechanics and Engineering 71 197-224

4. Silva, E. C. N. (1998) Design of Piezocomposite Materials and Piezoelectric Transducers Using Topology Optimization, Ph.D Dissertation, The University of Michigan-Ann Arbor.

5. Nishiwaki, S. (1998) Optimum Structural Topology Design Considering Flexibility, Ph.D Dissertation, The University of Michigan-Ann Arbor.

6. Kikuchi, N., Hollister, S. and Yoo, J. (1997) A Concept of Image-Based Intergrated CAE for Production -Engineering Proceedings of International Symposium on Optimization and Innovative Design Japan 75-90 